\title{
Active Cooling and Strain-Ratios to Increase the Actuation Frequency of SMA Wires
}

\author{
A. Lara-Quintanilla ${ }^{1}$ H. E. N. Bersee ${ }^{1}$
}

Published online: 28 October 2015

(C) ASM International 2015

\begin{abstract}
The maximum actuation frequency of an SMA wire does not only depend on the heating and cooling rates but also on the amplitude of strains at which it works. Smaller working amplitudes mean faster working frequencies. SMA-based actuators rarely work throughout their full strain range but they usually work at small amplitudes, which are amplified by means of mechanisms or by the optimised positioning of the wires within the actuator. Furthermore, the nonlinear relationship between the temperature and the strain on SMAs can be used in our favour to increase the actuation frequency of SMA wires by finding the optimum range of temperatures within which the wires must work for a specific strain amplitude. This work presents a method to find the maximum attainable actuation frequency of an SMA wire that is Joule heated and actively cooled while performing at different strainratios. The experimental results show that the actuation frequency of an SMA wire can be increased up to three and a half-fold (from 0.8 to $3 \mathrm{~Hz}$ ) just by making it work within the most appropriate range of temperatures, under similar heating and cooling rate, wire's diameter and applied stress.
\end{abstract}

Keywords Thermo-mechanical behaviour - Shape memory alloy $\cdot$ Stress-strain $\cdot$ Actuation frequency

A. Lara-Quintanilla

a.laraquintanilla@gmail.com

1 Structural Integrity and Composites, Faculty of Aerospace Engineering, Delft University of Technology, Delft, The Netherlands

\section{Introduction}

Shape memory alloy (SMA) wires are used to develop novel actuators. They can exert linear forces and strokes of up to $8 \%$ of strain and possesses a high power density [1, 2]. These characteristics make them attractive for actuation where limited room and weight are critical design requirements (e.g. in aerospace applications). However, the low actuation frequency of SMAs is a well-known limitation. It comes from the fact that they are thermally activated and, therefore, the time that it takes for the wire to contract and elongate strongly depends on the rate at which the wire is heated up (contraction time) and cooled down (cooling time), respectively. An SMA wire can be heated in different manners, although the most commonly used way is Joule heating due to its high efficiency. Depending on the application, the SMA wires can be passively or actively cooled. SMA wires are passively cooled when they release heat to the surrounding environment by conduction, convection or both without the existence of a system that controls the cooling rate of the wires [3]. Conversely, SMA wires are actively cooled when a system controls and increases the cooling rate of the SMA, thus increasing its actuation frequency $[4,5]$. The difference between the physics behind the heating and the cooling processes makes the contraction and the cooling times notably different. The cooling time is noticeably larger than the contraction time on SMA wires and, therefore, it is the limiting factor in terms of attainable actuation frequency. Some research has been carried out in order to increase the actuation frequency of SMA wires. There are several studies that focus on the improvement of the contraction time. For instance, Qiu et al. [6] used a circuit based on capacitors to accelerate the heating of SMA wires. Hunter et al. [7] applied large pulses of electric current. However, 
most of the research related to actuation frequency of SMAs focuses on the reduction of the cooling time since it is the limiting factor. Luchetti et al. [8] embedded an SMA wire into a silicone sleeve, reducing the cooling time of the wire by increasing its heat loss by conduction. Huang et al. embedded an SMA wire into a highly thermally conductive material [9]. Heat sink systems based on the Peltier effect have also shown to decrease the cooling time of SMA wires [10-12]. Tadesse et al. [13] compared the cooling times achieved under different cooling methods. These were, ordered from the least to most efficient, free convection, low-speed air, forced high-speed air and fluid quenching. The use of thermal gel was also tested but no contraction was observed on the SMA wire during the heating phase due to the very high thermal conductivity of the gel, which prevented the wire to heat up. The prestrain and the stress applied to the wires also influenced those times and, therefore, the ultimate actuation frequency of SMA-based actuators [13].

The work presented in this paper is related to the SMA-based actuator presented by Lara-Quintanilla et al. in [14]. This actuator has SMA wires placed in empty channels that go along the length of a thermoplastic beam. These channels are off the neutral axis of the beam. Thus, the SMA wires bend the beam upon contraction. The wires are Joule heated and actively cooled by means of a controlled airflow at room temperature, which is passed through the mentioned channels, cooling the wires by convection. Different amounts of energy can be supplied to the wire (heating rate) or released from it (cooling rate) by applying different amounts of electric power or different airflows around the wire, respectively. This has an impact on the time that it takes for the wire to contract and elongate. These times were studied [15] for an SMA wire that contracted and elongated throughout its full strain range.

The full strain range of an SMA wire is defined as the strain range between the maximum contraction of the wire and its maximum elongation for a certain applied stress. However, a wire working throughout its full strain range is not always an application requirement. In some cases, it is preferable to work at higher frequencies and smaller amplitudes. The ultimate amplitude of an actuator can afterwards be amplified by placing the wires in distinct ways or by using mechanisms (at the expense of the ultimate force exerted by the actuator). For instance, in our actuator [14] the embedded SMA wires are placed at $1.25 \mathrm{~mm}$ from the neutral axis and work at ranges of strain about $0.5 \mathrm{~mm}$ of amplitude. This contraction produces deflections on the tip of the beam of $\pm 30 \mathrm{~mm}$. For this reason, it becomes important to study the maximum frequency that the SMA wire can attain when working at different strains.
In this paper, a novel approach to increase the actuation frequency of SMA-based actuators is presented. It is based on the nonlinearity of the relationship between the temperature and the strain of SMAs. The actuation frequency of an SMA wire cannot only be increased by reducing the range of strains at which it works, but this nonlinearity can be used to our advantage by making the wire work within a range of strains that requires minimum variations of temperature to achieve great variations of strain. Luo et al. [10] used the transition between the austenitic and the rhombohedral phase (R-phase) since the temperature hysteresis is smaller than that between austenitic and martensitic phases. They controlled the temperature of the SMA to keep the SMA wire performing within the desired range of temperatures. However, we consider that controlling the strain is easier and more practical than controlling the temperature. Moreover, the strain of the SMA wire is usually the controlled variable.

Throughout this paper, we refer to strain-ratio as the ratio between the limited peak-to-peak amplitude of an experiment and the full strain range of the SMA wire. Thus, this study aims to find the actuation frequency of an SMA wire when it performs at a $5,10,15 \%$, etc. of its full strain range (strain-ratio $0.05,0.10,0.15$, etc.). In addition, these strain-ratios have been tested at three different segments of the full strain range.

This study aims to find to what extent the actuation frequency of an SMA wire can be increased by making it work within the appropriate range of strains, without varying either the heating or the cooling rate.

\section{Experimental}

The aim of this experiment is to find the maximum frequency at which an SMA wire can perform when it works at different strain intervals within the full strain range. The strain-temperature relationship on SMAs is highly nonlinear. As a consequence, a similar supply of power produces different strain rates depending on the current strain state of the SMA wire. This means that, for example, a supply of $20 \mathrm{~W}$ during $3 \mathrm{~s}$ produces a different contraction on the wire depending on whether the wire is not contracted at all or it is already $3 \%$ contracted. Therefore, it is necessary to study the SMA's behaviour when it works at different levels of strain in order to find the range of strains at which the maximum efficiency is achieved in terms of attainable frequency.

The isobaric setup for this experiment is shown in Fig. 1. This setup was initially used to cycle the $0.4-\mathrm{mm}$ diameter SmartFlex SMA wire [SAES Getters] at a constant stress of $234 \mathrm{MPa}$ (3 kg) for 8000 cycles in order to avoid functional fatigue on the wire. This number of cycles 


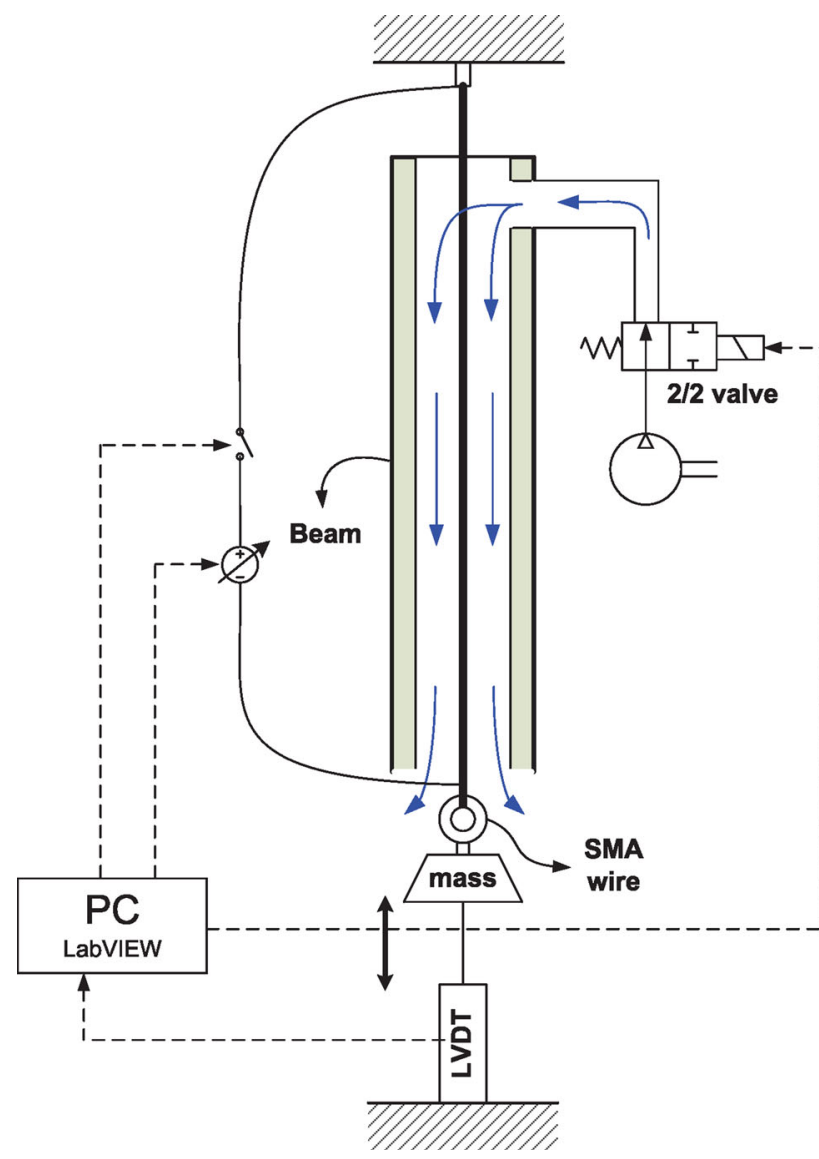

Fig. 1 Schematics of the isobaric setup

was experimentally verified to be sufficient to ensure wire stabilisation during the subsequent experiments (see [16] for further information about functional fatigue on SmartFlex SMA wires). Subsequently, a weight of $1 \mathrm{~kg}$ was hung from the wire. The SMA wire was placed into one of the channels of the beam since same heating and cooling conditions as those in real actuation wanted to be reproduced. The SMA wire was gripped to a fixed structure at the top. The electrical connections were made at both ends of the wire. A controlled power supply was used to supply electrical power to the wire $[\mathrm{SM} \sim 120-50 \sim \mathrm{DC}$ from Delta Elektronica BV]. The top end of the channel was covered and a hole of $0.4 \mathrm{~mm}$ was drilled on the cover to pass the wire through it while avoiding air leakage. The air inlet was on one side close to the top of the beam and the outlet was the bottom end of the channel. Thus, the airflow could go through the channel around the SMA wire, cooling it down. The amount of airflow allowed through the channel was controlled by a 2/2-way proportional valve [Type 2826 from Bürkert Fluid Control Systems] with the airflow at room temperature. The strain was calculated from the measurement of the linear variable differential transformer (LVDT), which measured the vertical

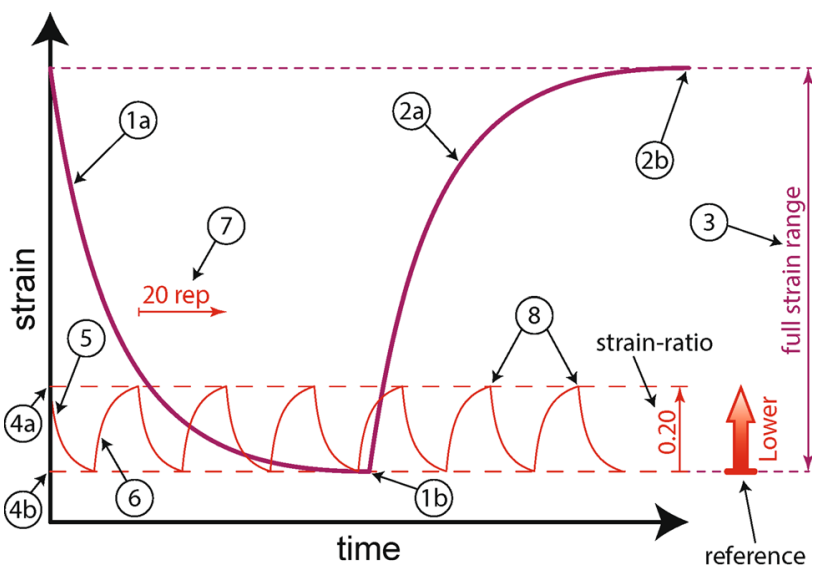

Fig. 2 Example of the procedure followed during each one of the experiments. Particularly in this example, a strain-ratio of 0.2 is tested taking the lower reference. The numbers in the figure correspond with the step number from the procedure

displacement of the hanging weight and, therefore, the contraction and elongation of the wire. The control of the power supply and the valve, as well as the data recording, was done by a computer running LabVIEW [National Instruments Corporation, Austin, TX, USA]. The analogueto-digital and the digital-to-analogue converters were National Instruments NI 9263 output module and 9215 input module connected to a NI cDAQ 9172 board. The control loop was executed at $50 \mathrm{~Hz}$ as well as the data recording (Fig. 1).

In a previous study, Lara-Quintanilla and Bersee [15] analysed the effect of applying different heating and cooling rates on the wire's response time. In the present work, as the maximum actuation frequency needed to be determined, only the maximum heating rate $(40 \mathrm{~W})$ and the maximum cooling rate $(24.1 \mathrm{l} / \mathrm{min})$ tested on the previous study were applied to the wire here. The wire was forced to work within a delimited range of strains. The actuation frequency $f_{\mathrm{a}}$ was calculated from the time that it took for the wire to contract $t_{\mathrm{h}}$ (contraction time) and elongate $t_{\mathrm{c}}$ (cooling time) as

$f_{\mathrm{a}}=\frac{1}{t_{\mathrm{h}}+t_{\mathrm{c}}}$.

The procedure followed by this test and its subsequent analysis is represented by Fig. 2. and described below:

Test procedure with LabVIEW

1. Heat the wire at $1.5 \mathrm{~A}$ for $20 \mathrm{~s}$. This ensures that the maximum contraction is reached. The lower limit of the full strain range is found.

2. Cool the wire at the maximum airflow $24.1 \mathrm{l} / \mathrm{min}$ for $20 \mathrm{~s}$. This ensures that the maximum elongation is reached. The upper limit of the full strain range is found. 
3. The strain between the lower and the upper limits is the full strain range.

4. Determine the upper and lower strain limits for the current test. This values depends on the strain-ratio of the current test and the reference that is taken (lower, middle and upper references are explained below).

5. Heat the wire at the maximum power $(40 \mathrm{~W})$ until the strain reaches the lower strain limit defined in 4 .

6. Cool the wire at the maximum opening of the valve (24.1 1/min) until the strain reaches the upper strain limit defined in 4.

7. Repeat the Steps 5 and 6 alternatively for 20 times (end of the test).

Test procedure with MATLAB

8. Detect the upper peaks on the strain time series.

9. Calculate time interval between consecutive upper peaks and average them. It is the sum of $t_{\mathrm{h}}$ (time interval from a peak to the next valley) and $t_{\mathrm{c}}$ (time interval from a valley to the next peak).

10. The time calculated at Step 9 is the inverse of $f_{\mathrm{a}}$. Calculate $f_{\mathrm{a}}$ (Eq. 1)

11. Repeat from Steps 4 to 10 for the different values of strain-ratio going from 0.05 to 1.00 ( $5 \%$ to the $100 \%$ of the full strain range) in increments of 0.05 .

The procedure described above was followed 3 times. In the first case (lower reference), the minimum strain (maximum contraction of the wire) was taken as reference. This reference was the lower limit. Since twenty strainratios were tested (Step 11), the upper limit of strain was modified for each test while keeping the lower limit constant. In the second case (middle reference), the middle value of strain between the maximum and the minimum ones was taken as reference. Both the upper and the lower limits were modified for each test and were at an equal distance from the reference. In the third case (upper reference), the maximum strain (maximum elongation of the wire) was taken as reference. This reference was the upper limit. Similarly to the lower reference case, the lower limit was modified for each test while keeping the upper limit constant. The procedure is shown in Fig. 3a.

As clarification, an example of the followed procedure is given: First, the wire with a hanging weight is heated until it reaches its maximum contraction, which is the lower level ( $0 \mathrm{~mm}$ of strain). Subsequently, the wire is cooled to room temperature, reaching its maximum elongation, which is the upper level (4 $\mathrm{mm}$ of strain). Therefore, the middle level is $2 \mathrm{~mm}$ of strain. These are the reference values. The first step is to calculate the limits for each strain-ratio. For example, in the case of strain-ratio 0.05 , the amplitude of the experiment must be $0.2 \mathrm{~mm}(0.05 \times 4 \mathrm{~mm}=0.2 \mathrm{~mm})$. Therefore, the limits (a)
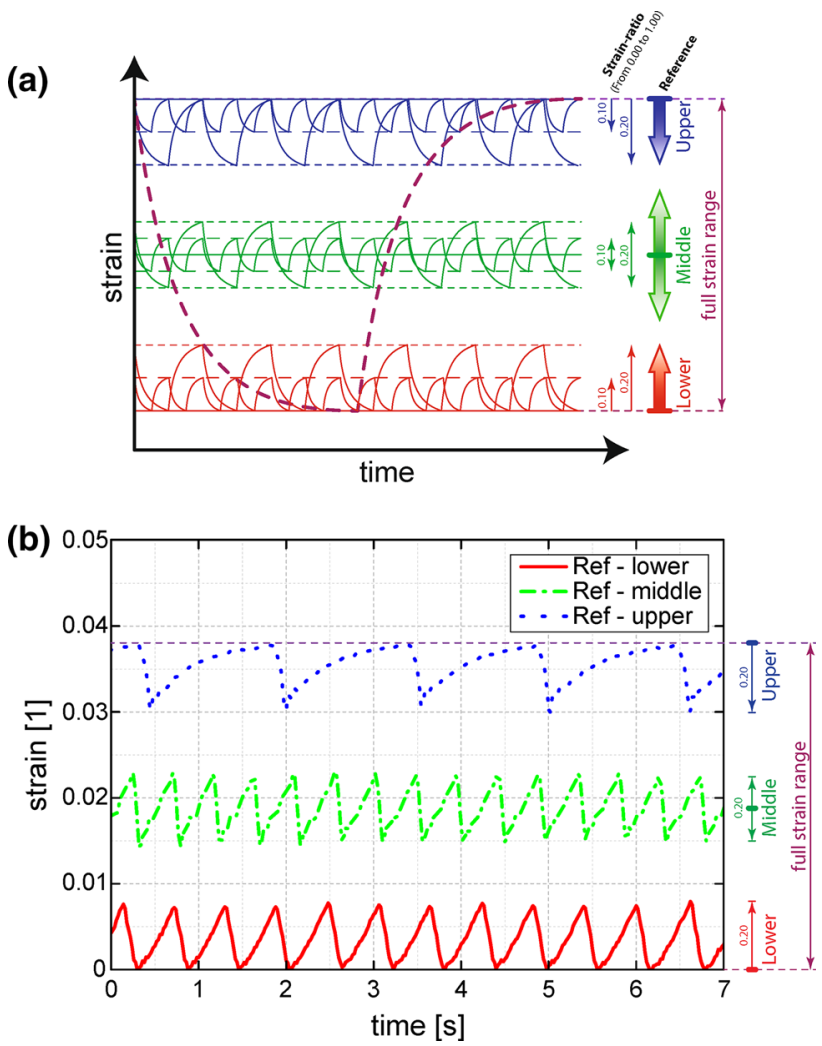

Fig. 3 Procedure to limit the strain of the wire at different references and strain-ratios. Explanatory diagram (a) and experimental data showing the strain time series corresponding to a 0.4 -mm-diameter SMA wire with a hanging weight of $1 \mathrm{~kg}$ working at a strain-ratio 0.20 for the three tested references (b)

of the lower reference test are 0 and $0.2 \mathrm{~mm}(0 \mathrm{~mm}$ is the reference), the limits of the middle reference test are 1.9 and $2.1 \mathrm{~mm}(2 \mathrm{~mm}$ is the reference) and the limits of the upper reference test are 3.8 and $4 \mathrm{~mm}$ ( $4 \mathrm{~mm}$ is the reference).

\section{Results and Discussion}

Four experiments were performed following the procedure explained above. Two wires of different diameters $(0.4$ and $0.2 \mathrm{~mm}$ ) were tested under two different loads (78 and $156 \mathrm{MPa}$ for each tested diameter). First, the attainable actuation frequency for $0.4 \mathrm{~mm}$ and $78 \mathrm{MPa}$ is discussed. Then, the effect of the applied load and the diameter of the SMA wire on its actuation frequency is shown.

\section{Attainable Actuation Frequency}

There is a great difference between the frequencies that can be achieved depending on the strain interval within which the SMA wire works. Figure 5a shows the achievable frequencies at different strain-ratios. Overall, the highest 


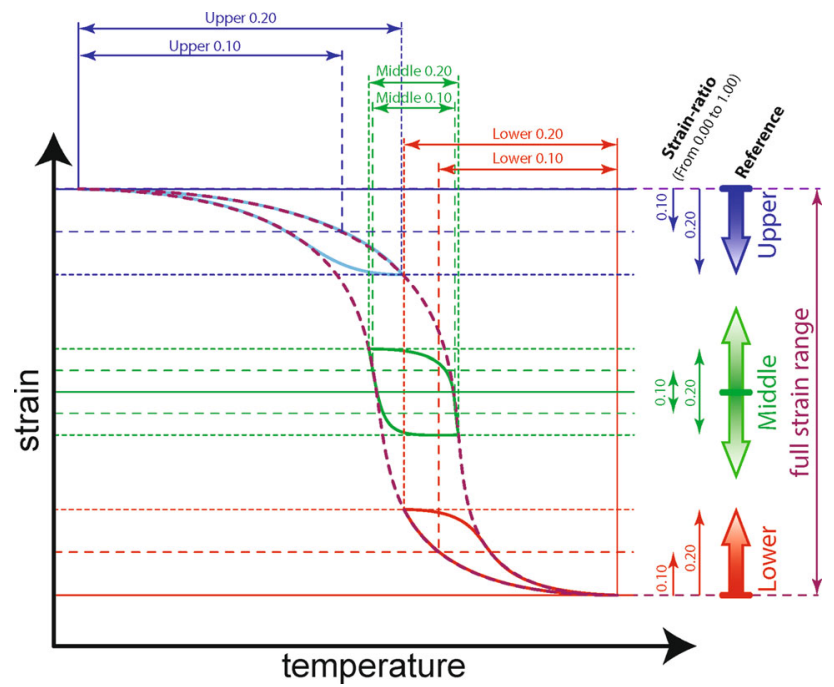

Fig. 4 Strain-temperature relationship of a SMA wire. Strain-ratios of 0.10 and 0.20 and their limits at different references are shown as an example. Upper reference: low temperature, maximum elongation. Middle reference: medium temperature, medium elongation. Lower reference: high temperature, maximum contraction. The bottom part of the graph shows how similar strain-ratios (on the right hand) can be attained at different intervals of temperature

frequencies are reached when the wire works around the middle reference, closely followed by the lower. The upper test reached much lower frequencies. In all the cases, the achievable frequency decreases as the strain-ratio increases. The difference between the frequencies reached by the tests at different references is more evident at low strainratios. For instance, at strain-ratio 0.05 , the frequency reached by the middle test is $3,2.4 \mathrm{~Hz}$ by the lower test and $0.8 \mathrm{~Hz}$ by the upper test. The frequency achieved by the middle test is three times higher than those achieved by the upper test for values of strain-ratio up to 0.25 . For strainratios above 0.55 , the frequencies from the upper test are half of those from the middle and lower tests, which are fairly similar. The resulting frequency when strain-ratio is ' 1 ' yields the same result since the wire performs throughout the full strain range whatever the reference is (Fig. 3).

Two phenomena are responsible for these results. From the point of view of heat loss, the wire's actuation frequency depends on the rate at which its temperature increases by Joule effect and decreases by convection between the wire and the surrounding air. Heating the wire is a much faster process than cooling it. Moreover, the heat loss rate $\dot{q}$ is proportional to the difference of temperatures between the SMA $T_{\text {wire }}$ and the surrounding air $T_{\text {airflow }}$ (see Eq. 2).

$\dot{q}=-h A\left(T_{\text {wire }}-T_{\text {airflow }}\right)$
This explains why the wire performed faster during the middle and lower tests, i.e. when the wire works at middle and high temperatures, respectively. However, this does not explain why the middle test yields the highest actuation frequency instead of the lower test.

Apart from this, there is the highly nonlinear relationship between temperature and strain. As it is shown in the Fig. 4, the slope of the strain is higher at values around the middle of the full strain range and smaller at values of strain that are close to the maximum or minimum strain of the wire. This means that small fluctuations of temperature provoke great deformations on the SMA wire around the middle strain. The wire's deformations are related to the phase transformations of the SMA's grains between martensite and austenite [17]. When the upper tests are run, the maximum elongation (maximum strain) of the wire is taken as reference. The maximum elongation happens when the wire is at room temperature and, therefore, martensite is the only stable phase in the SMA wire.

The contraction of the wire happens when the grains convert to austenite. This requires a significant increase of temperature up to the austenite temperature (around $90{ }^{\circ} \mathrm{C}$ for SmartFlex [18]). This contraction time is relatively long. Likewise, when the lower tests are run, the maximum contraction (minimum strain) of the wire is taken as a reference, what happens at high temperatures above the austenite temperature. The elongation of the wire happens when the grains convert into martensite, what requires a significant decrease of temperature (down to $70{ }^{\circ} \mathrm{C}$ for SmartFlex [18]). This cooling time is much longer than the contraction time (see Fig. 5b-d). However, when the middle tests are run (at an intermediate temperature), both phases co-exist since both of them are sufficiently stable to exist throughout the whole wire. At this point, small increments of temperature results in the transformation of many martensite grains into austenite, thus provoking a rapid contraction of the wire. Similarly, a small decrement of temperature results in the transformation of many austenite grains into martensite, what elongates the wire rapidly. For these reasons, the wire performed faster during the middle test.

Figure $5 b-d$ reveal the importance of the contraction and the cooling times with respect to the results. Although the frequency decreases noticeably as the strain-ratio increases, the contraction time remains low whereas the cooling time significantly increases. This result clearly shows that Joule heating is a much faster process than active cooling for SMA wires. The final actuation frequency of the SMA wire is, therefore, mainly limited by its cooling rate. 

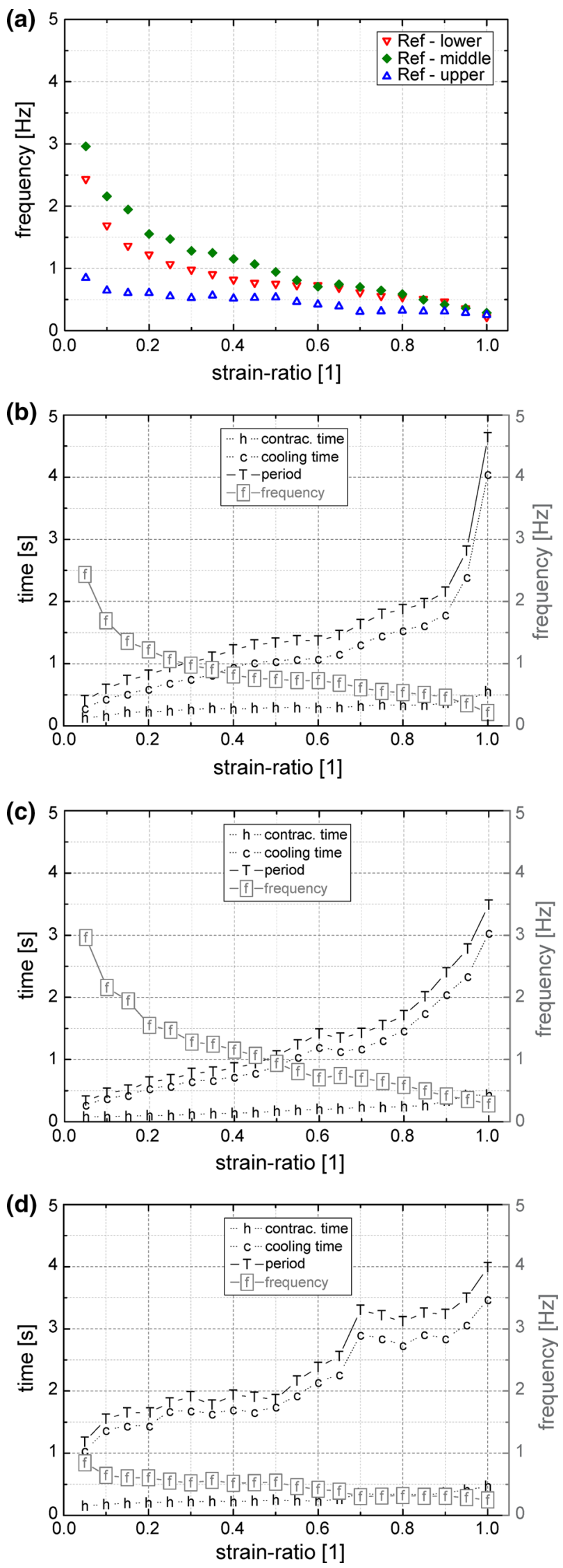

4Fig. 5 a Attainable frequencies at different strain-ratios and references for an applied load of $78 \mathrm{MPa}(1 \mathrm{~kg})$ on a 0.4 -mm-diameter SMA wire. The other graphs show the contraction and cooling times for the tested strain-ratios as well as the period (as the sum of the contraction and the cooling time) and the frequency (as the inverse of the period) reached by the lower reference test (b), middle reference test (c) and upper reference test (d)

\section{Effect of the Applied Load and the Diameter of the SMA Wire}

The wire's diameter and the applied load also influence the contraction and the cooling times and, as a result, the ultimate actuation frequency of the SMA wire. Higher loads entail shorter cooling times [15].

The same procedure described earlier was also followed with a weight of $2 \mathrm{~kg}$ on the $0.4-\mathrm{mm}$ SmartFlex wire. The results are shown in Fig. 6. The trends of the results are similar to those of the $1 \mathrm{~kg}$ test. The middle test reached the highest frequencies, followed by the lower test. The difference between the frequencies reached at each reference is more noticeable in the $2 \mathrm{~kg}$ test, and the frequencies reached by the middle and the lower tests only overlap from strain-ratio 0.80 onwards (Fig. 5).

In order to find the influence of the wire diameter on the resulting actuation frequency, the experiments were repeated using a $0.2-\mathrm{mm}$-diameter wire. In this case, weights of 0.25 and $0.5 \mathrm{~kg}$ were used because they exert the same stress on a $0.2-\mathrm{mm}$-diameter wire as the weights of 1 and $2 \mathrm{~kg}$ on a 0.4-mm-diameter wire $(78$ and $156 \mathrm{MPa}$, respectively), thus making the results comparable for a single variable, either the stress or the wire's diameter. The maximum power was also reduced to $10 \mathrm{~W}$ during the test on the $0.2-\mathrm{mm}$ wire in order to maintain the heating rate as $40 \mathrm{~W}$ on a 0.4 -mm-diameter wire. The airflow was maintained the same (24.1 1/min) (Fig. 6).

Figure 7 shows the reached frequencies by the middle reference tests for the two wire's diameters and stresses. The frequencies attained when doubling the load are slightly greater. The effect of the applied stress is more noticeable on the 0.4-mm-diameter wire. However, the diameter clearly plays a more important role. Under similar stress conditions, decreasing the wire's diameter significantly increases the actuation frequency. The working frequencies reached by the $0.2-\mathrm{mm}$-diameter wire are around two times higher than those reached by the $0.4-\mathrm{mm}$ diameter wire for all the tested strain-ratios except for 0.05 , a value for which it is slightly lower. The smaller the diameter is, the greater the surface-to-volume ratio of the wire is. Consequently, the heat losses by convection are also more significant, leading to a reduction of the cooling times (Fig. 7). 

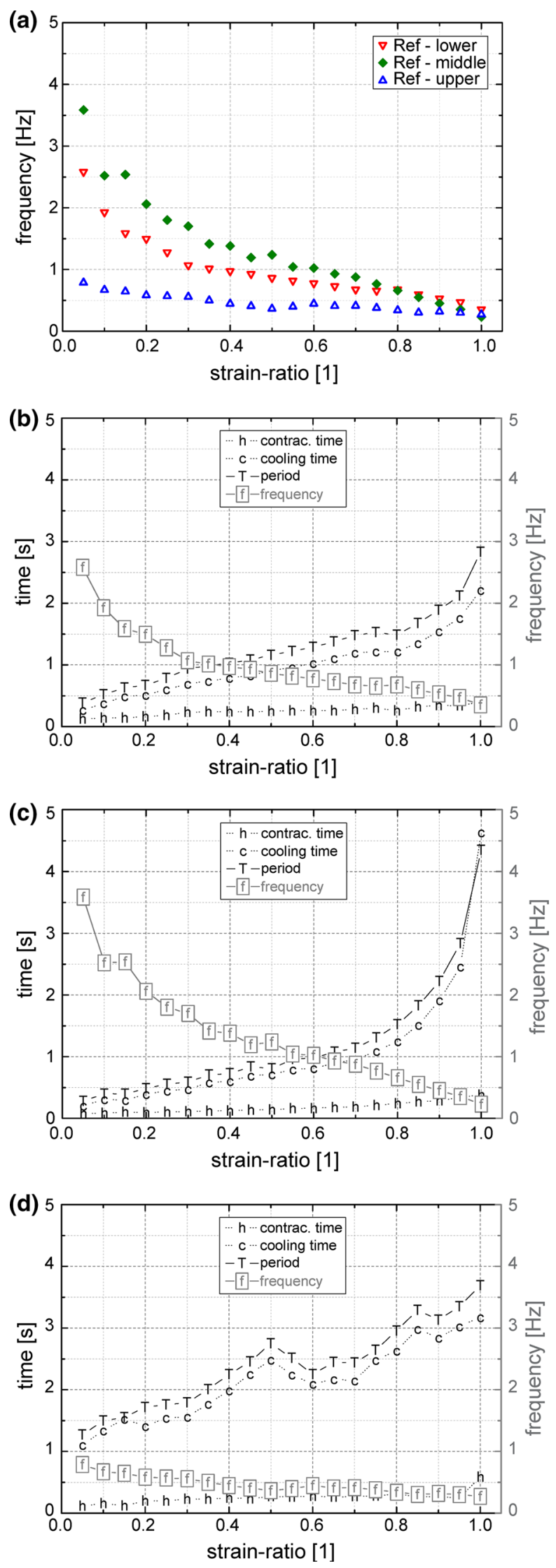

4Fig. 6 a Attainable frequencies at different strain-ratios and references for an applied load of $156 \mathrm{MPa}(2 \mathrm{~kg})$ on a $0.4-\mathrm{mm}$-diameter SMA wire. The other graphs show the contraction and cooling times for the tested strain-ratios as well as the period (as the sum of the contraction and the cooling time) and the frequency (as the inverse of the period) reached by the lower reference test (b), middle reference test (c) and upper reference test (d)

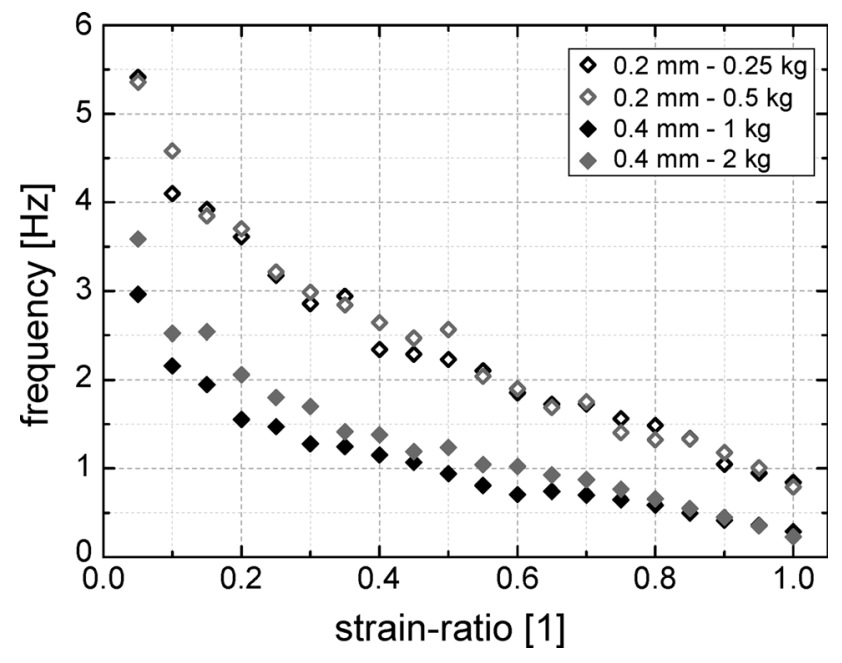

Fig. 7 Attainable frequencies reached by the middle reference test at different wire diameters and applied stresses

The heat losses-and, therefore, the wire's actuation frequency - can also be increased by lowering the temperature of the airflow or using a different fluid which offers a higher convective heat transfer coefficient $h$ (Eq. 2). Another option is to use an SMA wire whose transformation temperatures are higher, which increases the temperature difference between the wire and the cooling airflow, but this option is much less efficient from the energetic point of view given that the SMA wire should work at higher temperatures.

\section{Conclusions}

This paper proposes a method to find the maximum actuation frequency attainable by an SMA wire when it performs at different strain-ratios and at different strain ranges within its full strain range. In addition, SMA wires of 0.4 and $0.2 \mathrm{~mm}$ diameter have been tested under two different constant stresses in order to find the influence of the applied load and wire diameter on the maximum attainable frequency.

It is well-known that an SMA wire performs faster when the working amplitude is smaller under same heating and cooling conditions. However, the results from these experiments show that the attainable frequency does not 
only depend on the working amplitude but also on the interval of strains within which the wire works. It has been found that the best zone for the SMA to work is the interval of strains around the middle of the full strain range (middle test). The SMA's actuation frequency is three and a half times higher when it works around this zone than when it works around its maximum strain (upper test). The lower test, performed taking as reference the maximum contraction of the wire, reaches intermediate frequencies. In all the cases, the cooling times increase significantly as the tested strain-ratio increases, whereas the contraction time only slightly increases.

Two phenomena explain these results. From the thermodynamic point of view, the heat loss by convection is proportional to the difference of temperatures between the SMA wire and the surrounding airflow. This explains why the lower and middle tests reach higher actuation frequencies than the upper test. Apart from this, the nonlinear relationship between temperature and strain causes that small fluctuations of temperature that provoke great changes of strain on the SMA wire around the middle strain. This explains why the middle test reaches the higher actuation frequencies.

The applied load and the wire's diameter also have a certain influence on the attainable frequencies. Heavier loads have shown to slightly increase the actuation frequency of the wire. The influence of the applied load is more noticeable on the thicker wire. However, the wire's diameter plays a more important role on the attained frequency. The frequencies attainable by a 0.2 -mm-diameter wire are two times higher than those attained by a $0.4-\mathrm{mm}$ diameter wire. This happens because the heat loss by convection increases as the wire's diameter decreases.

This paper shows numerical results for a specific type of SMA wire (SmartFlex), diameter and applied load. However, similar conclusions in terms of attainable frequency, contraction and cooling times are expected for any other kind and size of SMA wires. Other proposed alternatives (not studied here) to increase the heat loss are the reduction of the temperature of the cooling airflow, the use of an SMA whose phase transformations occur at higher temperatures and the use of a fluid with a higher convective heat transfer coefficient.

The results and conclusions drawn here as well as the proposed method to find the maximum attainable frequency could hopefully be translatable to other smart materials in which their state variables are interrelated by nonlinear and hysteretic relationships.

Acknowledgments This work has been preformed within the framework of the European Community funded project Clean Sky
Joint Technology Initiative (JTI): 'Smart Fixed Wing Aircraft Integrated Technology Demonstrator' (SFWA-ITD).

Funding This research has been supported by CSJU-GAM-SFWA2008-001, within the European framework of JTI Clean Sky.

\section{References}

1. Pons JL (2005) Emerging actuator technologies: a micromechatronic approach. Wiley, New York

2. Callister WD, Rethwisch DG (2010) Materials science and engineering: an introduction, Eigth Binder Ready Version edn. Wiley, Hoboken

3. Gorbet RB, Morris KA, Chau RCC (2009) Mechanism of bandwidth improvement in passively cooled SMA position actuators. Smart Mater Struct 18(9):095013

4. Selden B, Cho K, Asada HH (2006) Segmented shape memory alloy actuators using hysteresis loop control. Smart Mater Struct 15(2):642

5. Gorbet RB, Russell RA (1995) A novel differential shape memory alloy actuator for position control. Robotica 13(04):423-430

6. Qiu J, Tani J, Osanai D, Urushiyama Y, Lewinnek D (2000) High-speed response of SMA actuators. Int J Appl Electromagn Mech 12(1):87-100

7. Hunter IW, Lafontaine S, Hollerbach JM, Hunter PJ (1991) Fast reversible NiTi fibers for use in microrobotics. In: Paper presented at the proceedings. IEEE Micro Electro Mechanical Systems

8. Luchetti T, Zanella A, Biasiotto M, Saccagno A (2009) Electrically actuated antiglare rear-view mirror based on a shape memory alloy actuator. J Mater Eng Perform 18(5-6):717-724

9. Huang S, Leary M, Ataalla T, Probst K, Subic A (2012) Optimisation of Ni-Ti shape memory alloy response time by transient heat transfer analysis. Mater Des 35:655-663

10. Luo Y, Takagi T, Maruyama S, Yamada M (2000) A shape memory alloy actuator using peltier modules and R-phase transition. J Intell Mater Syst Struct 11(7):503-511

11. Shahin AR, Meckl PH, Jones JD, Thrasher MA (1994) Enhanced cooling of shape memory alloy wires using semiconductor "heat pump" modules. J Intell Mater Syst Struct 5(1):95-104

12. Abadie J, Chaillet N, Lexcellent C (2002) An integrated shape memory alloy micro-actuator controlled by thermoelectric effect. Sens Actuators A 99(3):297-303

13. Tadesse Y, Thayer N, Priya S (2010) Tailoring the response time of shape memory alloy wires through active cooling and prestress. J Intell Mater Syst Struct 21(1):19-40. doi:10.1177/ 1045389x09352814

14. Lara-Quintanilla A, Hulskamp AW, Bersee HEN (2013) A highrate shape memory alloy actuator for aerodynamic load control on wind turbines. J Intell Mater Syst Struct 24(15):1834-1845

15. Lara-Quintanilla A, Bersee HE (2015) A study on the contraction and cooling times of actively cooled shape memory alloy wires. J Intell Mater Syst Struct. doi:10.1177/1045389x15595124

16. Scire-Mammano G, Dragoni E (2014) Functional fatigue of Ni-Ti shape memory wires under various loading conditions. Int $\mathrm{J}$ Fatigue 69:71-83

17. Lagoudas DC (2008) Shape memory alloys: modeling and engineering applications. Springer, New York

18. Hulskamp AW (2011) The smart rotor concept on wind turbines. Delft University of Technology, The Netherlands 